\title{
Ecological Risk Assesment and Seasonal-Spatial Distribution of Trace Elements in the Surface Sediment of Trabzon Harbour, Turkey
}

\author{
Y. Koray Kucuk ${ }^{1}$, Akasya Topcu ${ }^{2 *}$ \\ ${ }^{1}$ Ministry of Transport, Maritime Affairs and Communications, Ankara, Turkey \\ ${ }^{2}$ Department of Aquaculture and Fisheries Engineering, Agricultural Faculty, Ankara University, Ankara, Turkey \\ Email: *atopcu@ankara.edu.tr
}

How to cite this paper: Kucuk, Y.K. and Topcu, A. (2017) Ecological Risk Assesment and Seasonal-Spatial Distribution of Trace Elements in the Surface Sediment of Trabzon Harbour, Turkey. Open Journal of Ecology, 7, 348-363.

https://doi.org/10.4236/oje.2017.75025

Received: April 20, 2017

Accepted: May 23, 2017

Published: May 26, 2017

Copyright ( 92017 by authors and Scientific Research Publishing Inc. This work is licensed under the Creative Commons Attribution International License (CC BY 4.0).

http://creativecommons.org/licenses/by/4.0/

\section{Abstract}

A seasonal-spatial distribution and ecological risk assesment of the surface sediment are provided for Trabzon Harbour, located in the Eastern Black Sea Region. Sediments were collected from three stations, one of which was a control station, and a total of nine heavy metals were analyzed. In the sediment samples, concentrations of iron (Fe: $57.972 \pm 1.226-116.250 \pm 0.554$ $\left.\mu \mathrm{g} \cdot \mathrm{g}^{-1} \mathrm{DW}\right)$, copper (Cu: $\left.5.790 \pm 0.250-14.770 \pm 0.270 \mu \mathrm{g} \cdot \mathrm{g}^{-1} \mathrm{DW}\right)$, cadmium $\left(\mathrm{Cd}: 0.070 \pm 0.016-0.232 \pm 0.005 \mu \mathrm{g}^{-1} \mathrm{~g}^{-1} \mathrm{DW}\right)$, lead $(\mathrm{Pb}: 55.100 \pm 0.540-4.652$ $\pm 0.066 \mu \mathrm{g} \cdot \mathrm{g}^{-1} \mathrm{DW}$ ), chrome (Cr: $9.232 \pm 0.046-28.640 \pm 0.377 \mu \mathrm{g} \cdot \mathrm{g}^{-1} \mathrm{DW}$ ), zinc (Zn: $4.592 \pm 0.300-54.322 \pm 0.437 \mu \mathrm{g} \cdot \mathrm{g}^{-1} \mathrm{DW}$ ), arsenic (As: $2.702 \pm 0.233$ - $\left.6.332 \pm 0.186 \mu \mathrm{g} \cdot \mathrm{g}^{-1} \mathrm{DW}\right)$, manganese $(\mathrm{Mn}: 21.175 \pm 0.374-41.465 \pm 0.410$ $\mu \mathrm{g} \cdot \mathrm{g}^{-1} \mathrm{DW}$ ), nickel (Ni: $\left.9.272 \pm 0.042-54.230 \pm 0.158 \mu \mathrm{g} \cdot \mathrm{g}^{-1} \mathrm{DW}\right)$, total phosphorus (TP: $0.160 \pm 0.003-0.250 \pm 0.001 \mu \mathrm{g} \cdot \mathrm{g}^{-1} \mathrm{DW}$ ), total nitrogen (TN: $\left.0.111 \pm 0.001-0.161 \pm 0.001 \mu \mathrm{g} \cdot \mathrm{g}^{-1} \mathrm{DW}\right)$, organic matter $(\mathrm{OM}: 0.767 \pm 0.010-$ $1.750 \pm 0,009 \mu \mathrm{g} \cdot \mathrm{g}^{-1} \mathrm{DW}$ ), total organic carbon (TOC: $1.450 \pm 0.001-4.407 \pm$ $\left.0.002 \mu \mathrm{g} \cdot \mathrm{g}^{-1} \mathrm{DW}\right)$, clay $(10.845 \pm 0.347-40.545 \pm 0.830 ; \%)$, sand $(25.330 \pm$ $0.700-58.237 \pm 0.047 ; \%)$, and silt $(17.180 \pm 0.289-41.990 \pm 0.116$; \%) contents were determined. Dissolved oxygen $\left(\mathrm{O}_{2}: 9.100 \pm 0.115-14.530 \pm 0.047\right.$ $\left.\mathrm{mg} \cdot \mathrm{L}^{-1}\right)$, water temperature $\left(9.025 \pm 0.095-24.975 \pm 0.051^{\circ} \mathrm{C}\right), \mathrm{pH}(8.130 \pm$ $0.047-8.905 \pm 0.041$ ), and electrical conductivity (EC: $756.242 \pm 0.050-$ $780.655 \pm 0.213 \mathrm{mmhos} \cdot \mathrm{cm}^{-1}$ ) were measured in the sampling stations. Based on the concentration relationships, the SQGs (practical, reliable and predictive tools for assessing sediment quality), enrichment factor (EF) and Igeo analyses, the results indicated that the harbour has been contaminated by heavy metals to varying degrees, and organic material plays a key role in controlling the distribution of these heavy metal concentrations in the sediment. The spatial distribution pattern of heavy metals in the surface sediments of Trabzon Harbour is a basis for undertaking and providing monitoring studies 
of heavy metal contamination with regard to marine traffic, river input and anthropogenical effects around the port.

\section{Keywords}

Heavy Metals, Sediment, Sediment Quality Guidelines, EF, Igeo, Trabzon Harbour

\section{Introduction}

Sediments in aquatic ecosystems are home to many benthic and epibenthic organisms. They also affect the behavior of chemicals in these ecosystems. Moreover, they host and act as an important nutrient source for aquatic organisms, which in turn have an important effect on the pollution patterns in the aquatic systems. Sediments in marine environments serve as the ultimate sink of heavy metals [1]. They are also important for the transport and storage of potentially hazardous metals [2]. These metals enter into the aquatic system as a consequence of soil erosion, weathering of rocks, volcanic eruptions and human activities such as mining, dredging, and metal processing and use. Sediment analysis has an important part in determining the pollution status of marine environments [3].

Trace metals in aquatic environments accumulate primarily in the upper sections of sediment due to biological-geochemical mechanisms. These metals are toxic to marine organisms, and may cause death, impaired growth and reproduction capacity, and thus result in lower diversity of species [4] [5]. These metals also occur naturally in rock and can be introduced into aquatic environments through natural processes [6]. When heavy metals are present in aquatic ecosystems in concentrations exceeding the natural background load, they may accumulate to toxic levels without visible signs. This has become a problem of increasing concern.

It is important to determine whether sediment heavy metal concentrations pose a threat to aquatic organisms. The extent of metal pollution is determined by comparing metal concentrations in surface sediments to the TEL (threshold effects level) and PEL (probable effects level) of the SQGs (Sediment Quality Guidelines) established by [7] [8] and [9]. The extent of metal pollution in marine sediment can be assessed in a number of ways, such as USEPA, Igeo and Enrichment Factor (EF) [10] [11] [12] [13].

According to Turkey's foreign trade transport data from $2009,72 \%$ of the country's export and $94 \%$ of its import goods were transported by sea. Therefore, marine transportation is an important dynamic not only for the global economy, but also for Turkey's economy. The shipping trade is developing rapidly in parallel to advances in technology. In addition, as it is a safer and more economic means of transport, maritime transport is gradually increasing. However, these developments in maritime transport also bring about problems such 
as marine pollution caused by ships. The discharge of oil to the sea, the use of anti-fouling and anti-corrosive paints, oil spills during shipping and terminal transfers and effluent discharges from refineries probably comprise the anthropogenic sources of $\mathrm{Pb}, \mathrm{Cr}, \mathrm{Fe}, \mathrm{Cu}, \mathrm{Zn}$, and $\mathrm{Cd}$ into the oceans. Major ecological impacts have also increased in areas remote from the shoreline.

Although there are limited number of studies regarding heavy metal concentrations in the sediment of the Black Sea coastline [14] [15] [16] [17], to date there has been no research revealing the anthropogenic effects of metals and the environmental risk associated with their concentrations at Trabzon Harbour. The objectives of this research paper were to make the first records of: (1) the seasonal and spatial concentrations of the metals iron $(\mathrm{Fe})$, copper $(\mathrm{Cu})$, cadmium $(\mathrm{Cd})$, lead $(\mathrm{Pb})$, chromium $(\mathrm{Cr})$, zinc $(\mathrm{Zn})$, arsenic (As), manganese ( $\mathrm{Mn})$ and nickel (Ni) in the surface sediments of Trabzon Harbour; (2) the seasonal and spatial concentrations of the total phosphorus (TP), total nitrogen (TN), organic matter (OM), total organic carbon (TOC) with clay, sand and silt contents of the surface sediment; (3) the correlation between heavy metals and surface sediment chemical parameters; and (4) the environmental risk associated with the metal contents of the sediments using available SQGs.

\section{Materials and Methods}

\subsection{Site Description}

Trabzon is located on the coastline of the Black Sea, the world's largest inland sea, which has a link to the world's greatest seas and a place at the beginning of the transit route used by the Russian Federation and the Republics of Iran, Iraq, and Turkey. Trabzon Harbour plays a critical role in linking these countries to the European and world markets. Trabzon Harbour, having a length of $1.525 \mathrm{~m}$ docl, a three million ton storage capacity, a ship acceptance of 2.000 and a 250.000 passenger capacity, provides service to container ships, Ro-ro casting and shiploading [18]. Trabzon Harbour is located at $4057^{\prime} 30^{\prime \prime} \mathrm{N}-4106^{\prime} 36^{\prime \prime N}$ latitude and 40 02'30"E - 39 25'00"E longitude [19]. According to the Ship Trade General Management statistics for 2013, the number of sea vessels visiting Trabzon Harbour was 92 in January, 76 in February, 87 in March, 90 in April, 90 in May, 30 in June, 107 in July, 104 in August, 104 in September, 96 in October [20].

\subsection{Study Site}

The present research was conducted in Trabzon Harbour. Sediment and water samples were collected from three stations, of which the second (located in a dock with a depth of $9.5 \mathrm{~m}$ and a length of $400 \mathrm{~m}$ ) and third (located in a dock with a depth of $9.5 \mathrm{~m}$ and a length of $580 \mathrm{~m}$ ) stations in the port were selected to represent the Trabzon Harbour Maritime Authority, and the first station was the control station, located $1.530 \mathrm{~m}$ from the second and third stations (Figure 1). Sampling was carried out in April, July and October 2013 and January 2014, seasonally, with four renewals. 


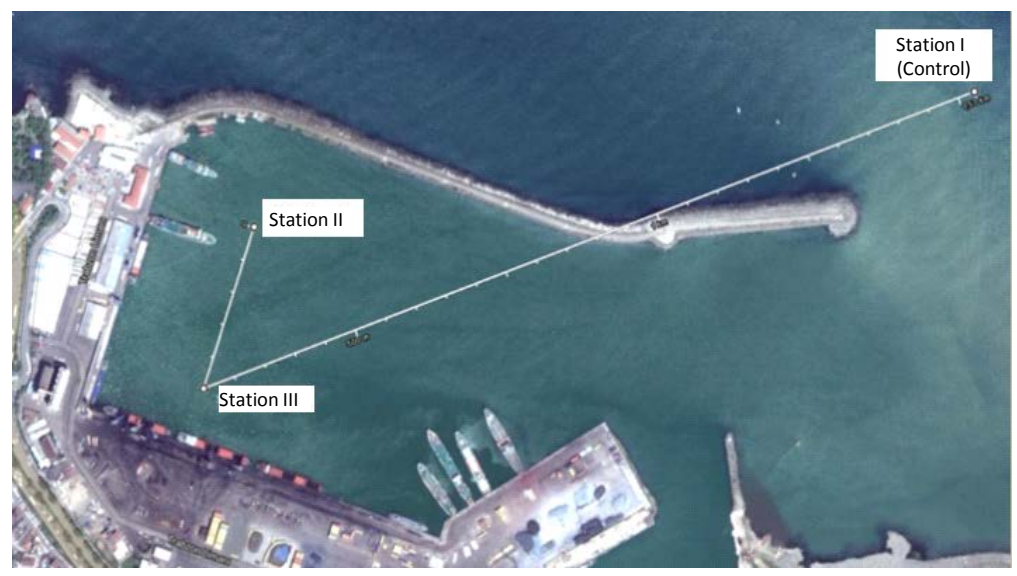

Figure 1. Trabzon Harbour and sampling stations.

\subsection{Sampling and in Situ Analyses}

Sediment samples were collected from the three stations using an Ekman-Birge grab and transferred to the laboratory in polyethylene bottles preserved in darkness to block the effect of sunlight. The dissolved oxygen and the temperature of the water were measured using an oxygen meter at the site. The $\mathrm{pH}$ of the water was estimated in situ with a $\mathrm{pH}$-meter.

Sediment samples were air-dried for a period of 20 days, homogenized by grinding, and finally passed through a $1.0 \mathrm{~mm}$ sieve for texture analyses in laboratory conditions. Organic matter (\%) was determined by the loss of weight during ignition at $550^{\circ} \mathrm{C}$ for 2 hours [21]. The water content of the sediment samples was specified by considering the difference between the samples before and after drying at $110^{\circ} \mathrm{C}$ for 16 hours according to [22]. Total organic carbon in the sediment samples was evaluated with an Organic Carbon Analyzer Unit where total nitrogen values were determined using the Dumas Method. Total phosphorus of the dried sediments was analysed spectrophotometrically after digestion in a mixture of oxidizing acids with reference to [21].

Surficial sediment samples for $\mathrm{Fe}, \mathrm{Cu}, \mathrm{Cd}, \mathrm{Pb}, \mathrm{Cr}, \mathrm{Zn}, \mathrm{As}, \mathrm{Mn}$, and $\mathrm{Ni}$ were dried at $60^{\circ} \mathrm{C}$ for 24 hours and then sieved and measured after wet digestion with an atomic absorption spectrophotometer (Perkin-Elmer 2380) operating on flame mode. The exchangeable fraction of metals was determined after extraction with a hydrofluoric/perchloric/sulfuric acid mixture. Detection of these metals was performed using the F-AAS technique [21].

\subsection{Statistical Analyses}

Statistical analyses were performed using Minitab and MStat software for Windows. ANOVA was used to evaluate the statistically significant differences between the sampling periods and sampling sites of the surface sediment [23].

\section{Results}

During the study, water temperature values at all stations ranged between 9.03 and $24.98^{\circ} \mathrm{C}$, while dissolved oxygen and $\mathrm{pH}$ values changed between 9.10 and 
$14.53 \mathrm{mg} \cdot \mathrm{L}^{-1}$ and 8.13 and 8.91 , respectively. The differences in mean EC values were found to be significant at all stations in all seasons $(p<0.05)$ (Table 1).

The differences in total phosphorus concentrations among the stations were found to be significant in all seasons $(p<0.05)$ except for the third station. The highest total phosphorus value $\left(0.25 \pm 0.003 \mu \mathrm{g} \cdot \mathrm{g}^{-1} \mathrm{DW}\right)$ was measured at the third station in January, whereas the lowest value $\left(0.16 \pm 0.003 \mu \mathrm{g} \cdot \mathrm{g}^{-1} \mathrm{DW}\right)$ was measured at the first station in April. The differences in total nitrogen concentrations between the seasons for the third station was found to be insignificant during the whole study period $(p>0.05)$, whereas the differences between the seasons for this parameter were found to be insignificant for the first and second stations except for January. The highest mean concentration of total nitrogen was found in April at the third station $\left(0.161 \pm 0.001 \mu \mathrm{g} \cdot \mathrm{g}^{-1} \mathrm{DW}\right)$. Total organic carbon values were between 1.45 and $4.41 \mu \mathrm{g} \cdot \mathrm{g}^{-1} \mathrm{DW}$ at all stations. Sediment TOC concentrations among the stations were found to be significant during the study period $(p<0.05)$ and the minimum value for this parameter was determined at the first station, whereas the maximum value was found at the third station. The differences in the sediment's mean LOI values between April 2013 and January 2014 were found to be statistically significant at all the stations for the entire research period $(p<0.05)$. In this study, water content values were between 56.86 and $68.70 \%$ (Table 2). The sediment texture values are shown in Table 2.

Table 1. Seasonal and spatial variations of water physico-chemical parametersin Trabzon Harbour (Mean value \pm standard deviation, $\mathrm{N}=4$ ).

\begin{tabular}{|c|c|c|c|c|}
\hline Parameters & $\begin{array}{l}\text { Stations } \\
\text { Months }\end{array}$ & I & II & III \\
\hline \multirow{4}{*}{$\begin{array}{c}\mathrm{O}_{2} \\
\left(\mathrm{mg} \cdot \mathrm{L}^{-1}\right)\end{array}$} & April 2013 & $11.700 \pm 0.091^{A^{*} c^{* *}}$ & $11.220 \pm 0.024^{\mathrm{Bc}}$ & $11.262 \pm 0.025^{\mathrm{Bc}}$ \\
\hline & July 2013 & $9.875 \pm 0.170^{\mathrm{Ad}}$ & $9.350 \pm 0.288^{\mathrm{Bd}}$ & $9.100 \pm 0.115^{\mathrm{Bd}}$ \\
\hline & October 2013 & $13.475 \pm 0.050^{\mathrm{Ab}}$ & $13.150 \pm 0.057^{\mathrm{Bb}}$ & $12.150 \pm 0.057^{\mathrm{Cb}}$ \\
\hline & January 2014 & $14.530 \pm 0.047^{\mathrm{Aa}}$ & $13.475 \pm 0.050^{\mathrm{Ba}}$ & $12.975 \pm 0.095^{\mathrm{Ca}}$ \\
\hline \multirow{4}{*}{$\begin{array}{c}\text { Water } \\
\text { temperature } \\
\left({ }^{\circ} \mathrm{C}\right)\end{array}$} & April 2013 & $19.920 \pm 0.053^{\mathrm{Ab}}$ & $19.930 \pm 0.053^{\mathrm{Ab}}$ & $19.582 \pm 0.053^{\mathrm{Bb}}$ \\
\hline & July 2013 & $24.225 \pm 0.287^{\mathrm{Ba}}$ & $24.922 \pm 0.051^{\mathrm{Aa}}$ & $24.975 \pm 0.051^{\mathrm{Aa}}$ \\
\hline & October 2013 & $17.825 \pm 0.050^{\mathrm{Bc}}$ & $18.227 \pm 0.032^{\mathrm{Ac}}$ & $18.492 \pm 0.032^{\mathrm{Ac}}$ \\
\hline & January 2014 & $9.025 \pm 0.095^{\mathrm{Cd}}$ & $9.167 \pm 0.039^{\mathrm{Bd}}$ & $9.275 \pm 0.039^{\mathrm{Ad}}$ \\
\hline \multirow{4}{*}{$\mathrm{pH}$} & April 2013 & $8.420 \pm 0.021^{\mathrm{Ab}}$ & $8.337 \pm 0.047^{\mathrm{Bb}}$ & $8.337 \pm 0.025^{\mathrm{BC}}$ \\
\hline & July 2013 & $8.322 \pm 0.033^{\mathrm{Ab}}$ & $8.345 \pm 0.031^{\mathrm{Ab}}$ & $8.212 \pm 0.015^{\mathrm{Bb}}$ \\
\hline & October 2013 & $8.130 \pm 0.047^{\mathrm{Ac}}$ & $8.180 \pm 0.040^{\mathrm{Ac}}$ & $8.175 \pm 0.028^{\mathrm{Aa}}$ \\
\hline & January 2014 & $8.905 \pm 0.041^{\mathrm{Aa}}$ & $8.837 \pm 0.025^{\mathrm{Ba}}$ & $8.832 \pm 0.015^{\mathrm{Bd}}$ \\
\hline \multirow{4}{*}{$\begin{array}{c}\text { EC } \\
\left(\mathrm{mmhos} \cdot \mathrm{cm}^{-1}\right)\end{array}$} & April 2013 & $778.835 \pm 1.150^{\mathrm{Ba}}$ & $780.410 \pm 0.430^{\mathrm{Aa}}$ & $780.655 \pm 0.213^{\mathrm{Aa}}$ \\
\hline & July 2013 & $757.252 \pm 1.349^{A c}$ & $756.765 \pm 0.231^{\mathrm{Ad}}$ & $756.242 \pm 0.050^{\mathrm{Ad}}$ \\
\hline & October 2013 & $761.250 \pm 0.957^{\mathrm{Ab}}$ & $761.250 \pm 1.50^{\mathrm{Ac}}$ & $761.250 \pm 0.50^{\mathrm{Ac}}$ \\
\hline & January 2014 & $756.882 \pm 0.174^{\mathrm{Cd}}$ & $769.337 \pm 0.942^{\mathrm{Bb}}$ & $774.540 \pm 0.50^{\mathrm{Ab}}$ \\
\hline
\end{tabular}

*The different upper-case letters in the same row show the differences between stations $(p<0.05)$, ${ }^{*}$ The different lower-case letters in the same column show the differences between months $(p<0.05)$ 
Table 2. Seasonal and spatial variations of sediment organic material (OM), total organic carbon (TOC), total phosphorus (TP), total nitrogen (TN), water content and sediment texture in Trabzon Harbour (Mean value \pm standard deviation, $\mathrm{N}=4$ ).

\begin{tabular}{|c|c|c|c|c|}
\hline Parameters & $\begin{array}{l}\text { Stations } \\
\text { Months }\end{array}$ & I & II & III \\
\hline \multirow{4}{*}{$\begin{array}{c}\mathrm{OM} \\
\left(\mu \mathrm{g} \cdot \mathrm{g}^{-1} \mathrm{DW}\right)\end{array}$} & April 2013 & $0.902 \pm 0.170^{C^{\star} b^{* *}}$ & $1.340 \pm 0.035^{\mathrm{Bb}}$ & $1.660 \pm 0.014^{\mathrm{Ab}}$ \\
\hline & July 2013 & $1.262 \pm 0.030^{\mathrm{Ca}}$ & $1.420 \pm 0.024^{\mathrm{Ba}}$ & $1.750 \pm 0.009^{\mathrm{Aa}}$ \\
\hline & October 2013 & $0.892 \pm 0.010^{\mathrm{Cb}}$ & $1.180 \pm 0.022^{\mathrm{Ac}}$ & $1.092 \pm 0.009^{\mathrm{Bd}}$ \\
\hline & January 2014 & $0.767 \pm 0.010^{\mathrm{Cc}}$ & $1.447 \pm 0.050^{\mathrm{Aa}}$ & $1.340 \pm 0.014^{\mathrm{Bc}}$ \\
\hline \multirow{4}{*}{$\begin{array}{c}\text { TOC } \\
\left(\mu \mathrm{g} \cdot \mathrm{g}^{-1} \mathrm{DW}\right)\end{array}$} & April 2013 & $1.500 \pm 0.004^{\mathrm{Cb}}$ & $2.198 \pm 0.002^{\mathrm{Bd}}$ & $4.407 \pm 0.002^{\mathrm{Aa}}$ \\
\hline & July 2013 & $1.850 \pm 0.002^{\mathrm{Ca}}$ & $3.114 \pm 0.002^{\mathrm{Ba}}$ & $4.225 \pm 0.002^{\mathrm{Ab}}$ \\
\hline & October 2013 & $1.450 \pm 0.001^{\mathrm{Cd}}$ & $2.400 \pm 0.002^{\mathrm{Bc}}$ & $2.520 \pm 0.002^{\mathrm{Ad}}$ \\
\hline & January 2014 & $1.464 \pm 0.002^{\mathrm{Cc}}$ & $2.530 \pm 0.007^{\mathrm{Bb}}$ & $2.720 \pm 0.003^{\mathrm{Ac}}$ \\
\hline \multirow{4}{*}{$\begin{array}{c}\text { TP } \\
\left(\mu g \cdot g^{-1} \mathrm{DW}\right)\end{array}$} & April 2013 & $0.160 \pm 0.003^{\mathrm{Bd}}$ & $0.220 \pm 0.002^{\mathrm{Ac}}$ & $0.220 \pm 0.001^{\mathrm{Ac}}$ \\
\hline & July 2013 & $0.184 \pm 0.001^{\mathrm{Cc}}$ & $0.210 \pm 0.002^{\mathrm{Bd}}$ & $0.220 \pm 0.001^{\mathrm{Ac}}$ \\
\hline & October 2013 & $0.210 \pm 0.001^{\mathrm{Cb}}$ & $0.223 \pm 0.002^{\mathrm{Bb}}$ & $0.230 \pm 0.001^{\mathrm{Ab}}$ \\
\hline & January 2014 & $0.214 \pm 0.001^{\mathrm{Ca}}$ & $0.241 \pm 0.001^{\mathrm{Ba}}$ & $0.250 \pm 0.001^{\mathrm{Aa}}$ \\
\hline \multirow{4}{*}{$\begin{array}{c}\text { TN } \\
\left(\mu \mathrm{g} \cdot \mathrm{g}^{-1} \mathrm{DW}\right)\end{array}$} & April 2013 & $0.122 \pm 0.001^{\mathrm{Cc}}$ & $0.131 \pm 0.009^{\mathrm{Bb}}$ & $0.161 \pm 0.009^{\mathrm{Aa}}$ \\
\hline & July 2013 & $0.120 \pm 0.009^{\mathrm{Cc}}$ & $0.128 \pm 0.006^{\mathrm{Bb}}$ & $0.156 \pm 0.002^{\mathrm{Aa}}$ \\
\hline & October 2013 & $0.112 \pm 0.001^{\mathrm{Cc}}$ & $0.124 \pm 0.001^{\mathrm{Bb}}$ & $0.137 \pm 0.006^{\mathrm{Aa}}$ \\
\hline & January 2014 & $0.145 \pm 0.011^{\mathrm{Aa}}$ & $0.145 \pm 0.010^{\mathrm{Aa}}$ & $0.141 \pm 0.001^{\mathrm{Aa}}$ \\
\hline \multirow{4}{*}{$\begin{array}{c}\text { Water Content } \\
(\%)\end{array}$} & April 2013 & $67.31 \pm 0.50^{\mathrm{Ab}}$ & $64.38 \pm 0.63^{\mathrm{Ba}}$ & $60.05 \pm 0.83^{\mathrm{Ca}}$ \\
\hline & July 2013 & $65.13 \pm 1.13^{\mathrm{Ac}}$ & $60.15 \pm 0.51^{\mathrm{Bb}}$ & $56.86 \pm 0.55^{\mathrm{Cb}}$ \\
\hline & October 2013 & $68.70 \pm 0.43^{\mathrm{Aa}}$ & $58.67 \pm 1.09^{\mathrm{BC}}$ & $57.72 \pm 0.41^{\mathrm{Bb}}$ \\
\hline & January 2014 & $67.60 \pm 0.49^{\mathrm{Ab}}$ & $59.10 \pm 0.44^{\mathrm{Bc}}$ & $59.37 \pm 0.73^{\mathrm{Ba}}$ \\
\hline \multirow{4}{*}{$\begin{array}{l}\text { Clay } \\
(\%)\end{array}$} & April 2013 & $13.412 \pm 0.123^{\mathrm{Cc}}$ & $30.330 \pm 0.472^{\mathrm{Bc}}$ & $35.317 \pm 0.407^{\mathrm{Ac}}$ \\
\hline & July 2013 & $17.590 \pm 0.416^{\mathrm{Ca}}$ & $24.830 \pm 0.578^{\mathrm{Bd}}$ & $37.422 \pm 0.179^{\mathrm{Aa}}$ \\
\hline & October 2013 & $14.940 \pm 0.463^{\mathrm{Cb}}$ & $35.730 \pm 0.552^{\mathrm{Ab}}$ & $31.980 \pm 0.749^{\mathrm{Bd}}$ \\
\hline & January 2014 & $10.845 \pm 0.347^{\mathrm{Cd}}$ & $40.545 \pm 0.830^{\mathrm{Aa}}$ & $36.305 \pm 0.375^{\mathrm{Bb}}$ \\
\hline \multirow{4}{*}{$\begin{array}{l}\text { Silt } \\
(\%)\end{array}$} & April 2013 & $35.920 \pm 0.204^{\mathrm{Bd}}$ & $25.105 \pm 0.392^{\mathrm{Cc}}$ & $37.812 \pm 0.245^{\mathrm{Aa}}$ \\
\hline & July 2013 & $39.800 \pm 0.576^{\mathrm{Ab}}$ & $17.180 \pm 0.289^{\mathrm{Cd}}$ & $37.485 \pm 0.969^{\mathrm{Ba}}$ \\
\hline & October 2013 & $39.155 \pm 0.370^{\mathrm{Ac}}$ & $38.110 \pm 0.408^{\mathrm{Ba}}$ & $30.972 \pm 0.713^{\mathrm{Cc}}$ \\
\hline & January 2014 & $41.990 \pm 0.116^{\mathrm{Aa}}$ & $37.010 \pm 0.619^{\mathrm{Bb}}$ & $33.502 \pm 0.278^{\mathrm{Cb}}$ \\
\hline \multirow{4}{*}{$\begin{array}{l}\text { Sand } \\
(\%)\end{array}$} & April 2013 & $51.452 \pm 1.025^{\mathrm{Aa}}$ & $45.302 \pm 0.106^{\mathrm{Bb}}$ & $27.345 \pm 0.065^{\mathrm{Cc}}$ \\
\hline & July 2013 & $42.675 \pm 0.452^{\mathrm{Bd}}$ & $58.237 \pm 0.047^{\mathrm{Aa}}$ & $25.292 \pm 0.042^{\mathrm{Cd}}$ \\
\hline & October 2013 & $46.690 \pm 0.638^{\mathrm{Ac}}$ & $26.502 \pm 0.415^{\mathrm{Cc}}$ & $37.337 \pm 0.594^{\mathrm{Ba}}$ \\
\hline & January 2014 & $47.750 \pm 0.091^{\mathrm{Ab}}$ & $25.330 \pm 0.700^{\mathrm{Cd}}$ & $29.715 \pm 0.507^{\mathrm{Bb}}$ \\
\hline
\end{tabular}

${ }^{*}$ The different upper-case letters in the same row show the differences between stations $(p<0.05)$, ${ }^{* *}$ The different lower-case letters in the same column show the differences between months $(p<0.05)$

The mean concentrations and standard deviations of the studied heavy metals in the sediment from Trabzon Harbour are presented in Table 3. Based on their maximum content, the components of the surface sediment were arranged in the following decreasing order: $\mathrm{Fe}>\mathrm{Pb}>\mathrm{Zn}>\mathrm{Ni}>\mathrm{Mn}>\mathrm{Cr}>\mathrm{Cu}>\mathrm{As}>\mathrm{Cd}$.

Significant positive and negative correlations between certain sediment chemical properties and heavy metals were established in the summer period, keeping 
in mind that generally, heavy metal toxicity has the greatest effect on aquatic organisms in summer months (Tables 4-6).

Table 3. Seasonal and spatial variations of sediment heavy metal concentrations in Trabzon Harbour (Mean value \pm standard deviation, $\mathrm{N}=4$ ).

\begin{tabular}{|c|c|c|c|c|}
\hline Parameters & $\begin{array}{l}\text { Stations } \\
\text { Months }\end{array}$ & I & II & III \\
\hline \multirow{5}{*}{$\begin{array}{c}\mathrm{Fe} \\
\left(\mu \mathrm{g} \cdot \mathrm{g}^{-1} \mathrm{DW}\right)\end{array}$} & April 2013 & $57.972 \pm 1.226^{\mathrm{C}^{*} \mathrm{c}^{* *}}$ & $116.250 \pm 0.554^{\mathrm{Aa}}$ & $101.800 \pm 0.481^{\mathrm{Bb}}$ \\
\hline & July 2013 & $71.040 \pm 0.581^{\mathrm{Ba}}$ & $110.170 \pm 0.750^{\mathrm{Ab}}$ & $111.017 \pm 0.793^{\mathrm{Aa}}$ \\
\hline & October 2013 & $56.600 \pm 0.921^{\mathrm{Cd}}$ & $95.815 \pm 0.370^{\mathrm{Ac}}$ & $82.762 \pm 0.913^{\mathrm{Bc}}$ \\
\hline & January 2014 & $61.942 \pm 0.467^{\mathrm{Cb}}$ & $96.160 \pm 0.550^{\mathrm{Ac}}$ & $82.915 \pm 0.853^{\mathrm{Bc}}$ \\
\hline & April 2013 & $6.180 \pm 0.730^{\mathrm{Cb}}$ & $13.000 \pm 0.046^{\mathrm{Bb}}$ & $14.437 \pm 0.520^{\mathrm{Aa}}$ \\
\hline \multirow{4}{*}{$\begin{array}{c}\mathrm{Cu} \\
\left(\mu \mathrm{g} \cdot \mathrm{g}^{-1} \mathrm{DW}\right)\end{array}$} & July 2013 & $7.102 \pm 0.311^{\mathrm{Ca}}$ & $10.520 \pm 0.581^{\mathrm{Bc}}$ & $14.017 \pm 0.160^{\mathrm{Aa}}$ \\
\hline & October 2013 & $5.790 \pm 0.258^{\mathrm{Cb}}$ & $14.740 \pm 0.300^{\mathrm{Aa}}$ & $11.447 \pm 0.358^{\mathrm{Bb}}$ \\
\hline & January 2014 & $6.050 \pm 0.150^{\mathrm{Cb}}$ & $14.770 \pm 0.270^{\mathrm{Aa}}$ & $14.222 \pm 0.255^{\mathrm{Ba}}$ \\
\hline & April 2013 & $0.120 \pm 0.082^{\mathrm{Cb}}$ & $0.152 \pm 0.095^{\mathrm{Bc}}$ & $0.232 \pm 0.005^{\mathrm{Aa}}$ \\
\hline \multirow{4}{*}{$\begin{array}{c}\mathrm{Cd} \\
\left(\mu \mathrm{g} \cdot \mathrm{g}^{-1} \mathrm{DW}\right)\end{array}$} & July 2013 & $0.074 \pm 0.001^{\mathrm{Cc}}$ & $0.112 \pm 0.050^{\mathrm{Bd}}$ & $0.142 \pm 0.005^{\mathrm{Ad}}$ \\
\hline & October 2013 & $0.160 \pm 0.005^{\mathrm{Ca}}$ & $0.230 \pm 0.082^{\mathrm{Aa}}$ & $0.177 \pm 0.005^{\mathrm{Bc}}$ \\
\hline & January 2014 & $0.070 \pm 0.016^{\mathrm{Cc}}$ & $0.170 \pm 0.050^{\mathrm{Bb}}$ & $0.192 \pm 0.005^{\mathrm{Ab}}$ \\
\hline & April 2013 & $4.652 \pm 0.066^{\mathrm{Cd}}$ & $6.442 \pm 0.160^{\mathrm{Ad}}$ & $5.702 \pm 0.102^{\mathrm{Bd}}$ \\
\hline \multirow{4}{*}{$\begin{array}{c}\mathrm{Pb} \\
\left(\mu \mathrm{g} \cdot \mathrm{g}^{-1} \mathrm{DW}\right)\end{array}$} & July 2013 & $6.625 \pm 0.119^{\mathrm{Cb}}$ & $12.325 \pm 0.087^{\mathrm{Bb}}$ & $14.245 \pm 0.052^{\mathrm{Aa}}$ \\
\hline & October 2013 & $7.212 \pm 0.164^{\mathrm{Ca}}$ & $10.275 \pm 0.130^{\mathrm{Ac}}$ & $8.640 \pm 0.126^{\mathrm{Bc}}$ \\
\hline & January 2014 & $5.420 \pm 0.024^{\mathrm{Cc}}$ & $55.100 \pm 0.540^{\mathrm{Aa}}$ & $11.822 \pm 0.026^{\mathrm{Bb}}$ \\
\hline & April 2013 & $9.232 \pm 0.046^{\mathrm{Bd}}$ & $13.372 \pm 0.204^{\mathrm{Ab}}$ & $9.422 \pm 0.120^{\mathrm{Bd}}$ \\
\hline \multirow{4}{*}{$\begin{array}{c}\mathrm{Cr} \\
\left(\mu \mathrm{g} \cdot \mathrm{g}^{-1} \mathrm{DW}\right)\end{array}$} & July 2013 & $11.892 \pm 0.197^{\mathrm{Bc}}$ & $16.100 \pm 4.985^{\mathrm{Bb}}$ & $28.640 \pm 0.377^{\mathrm{Aa}}$ \\
\hline & October 2013 & $12.395 \pm 0.031^{\mathrm{Cb}}$ & $12.780 \pm 0.059^{\mathrm{Ab}}$ & $12.602 \pm 0.020^{\mathrm{Bc}}$ \\
\hline & January 2014 & $16.205 \pm 0.117^{\mathrm{Ca}}$ & $20.050 \pm 0.067^{\mathrm{Aa}}$ & $17.212 \pm 0.165^{\mathrm{Bb}}$ \\
\hline & April 2013 & $4.592 \pm 0.300^{\mathrm{Cb}}$ & $27.572 \pm 0.300^{\mathrm{Bb}}$ & $54.322 \pm 0.437^{\mathrm{Aa}}$ \\
\hline \multirow{4}{*}{$\underset{\left(\mu \mathrm{g} \cdot \mathrm{g}^{-1} \mathrm{DW}\right)}{\mathrm{Zn}}$} & July 2013 & $4.925 \pm 0.080^{\mathrm{Cb}}$ & $20.262 \pm 0.470^{\mathrm{Bc}}$ & $39.372 \pm 0.314^{\mathrm{Ab}}$ \\
\hline & October 2013 & $4.902 \pm 0.127^{\mathrm{Cb}}$ & $29.950 \pm 0.210^{\mathrm{Aa}}$ & $27.090 \pm 0.344^{\mathrm{Bd}}$ \\
\hline & January 2014 & $5.365 \pm 0.300^{\mathrm{Ca}}$ & $29.470 \pm 0.453^{\mathrm{Ba}}$ & $30.710 \pm 0.423^{\mathrm{Ac}}$ \\
\hline & April 2013 & $2.762 \pm 0.101^{\mathrm{Bc}}$ & $2.702 \pm 0.233^{\mathrm{Bd}}$ & $3.527 \pm 0.305^{\mathrm{Ac}}$ \\
\hline \multirow{4}{*}{$\begin{array}{c}\text { As } \\
\left(\mu \mathrm{g} \cdot \mathrm{g}^{-1} \mathrm{DW}\right)\end{array}$} & July 2013 & $3.107 \pm 0.071^{\mathrm{Cb}}$ & $6.160 \pm 0.048^{\mathrm{Aa}}$ & $5.715 \pm 0.338^{\mathrm{Bb}}$ \\
\hline & October 2013 & $3.532 \pm 0.041^{\mathrm{Ca}}$ & $3.937 \pm 0.017^{\mathrm{Ab}}$ & $3.807 \pm 0.043^{\mathrm{Bc}}$ \\
\hline & January 2014 & $3.222 \pm 0.181^{\mathrm{Bb}}$ & $3.332 \pm 0.132^{\mathrm{Bc}}$ & $6.332 \pm 0.186^{\mathrm{Aa}}$ \\
\hline & April 2013 & $28.840 \pm 0.448^{\mathrm{Ba}}$ & $28.780 \pm 0.255^{\mathrm{Bc}}$ & $31.625 \pm 0.327^{\mathrm{Ab}}$ \\
\hline \multirow{4}{*}{$\underset{\left(\mu \mathrm{g} \cdot \mathrm{g}^{-1} \mathrm{DW}\right)}{\mathrm{Mn}}$} & July 2013 & $27.370 \pm 0.310^{\mathrm{Cb}}$ & $28.101 \pm 0.545^{\mathrm{Bd}}$ & $29.757 \pm 0.327^{\mathrm{Ac}}$ \\
\hline & October 2013 & $21.175 \pm 0.374^{\mathrm{Cc}}$ & $41.465 \pm 0.410^{\mathrm{Aa}}$ & $29.982 \pm 0.578^{\mathrm{Bc}}$ \\
\hline & January 2014 & $23.652 \pm 0.398^{\mathrm{Cb}}$ & $40.670 \pm 0.420^{\mathrm{Ab}}$ & $35.235 \pm 0.590^{\mathrm{Ba}}$ \\
\hline & April 2013 & $9.272 \pm 0.042^{\mathrm{Cd}}$ & $11.565 \pm 0.147^{\mathrm{Bd}}$ & $13.535 \pm 0.068^{\mathrm{Ac}}$ \\
\hline \multirow{3}{*}{$\begin{array}{c}\mathrm{Ni} \\
\left(\mu \mathrm{g} \cdot \mathrm{g}^{-1} \mathrm{DW}\right)\end{array}$} & July 2013 & $11.470 \pm 0.073^{\mathrm{Cc}}$ & $11.407 \pm 0.073^{\mathrm{Bb}}$ & $47.372 \pm 0.453^{\mathrm{Aa}}$ \\
\hline & October 2013 & $13.310 \pm 0.105^{\mathrm{Bb}}$ & $15.240 \pm 0.251^{\mathrm{Ac}}$ & $13.515 \pm 0.030^{\mathrm{Bc}}$ \\
\hline & January 2014 & $20.112 \pm 0.137^{\mathrm{Ca}}$ & $54.230 \pm 0.158^{\mathrm{Aa}}$ & $24.740 \pm 0.048^{\mathrm{Bb}}$ \\
\hline
\end{tabular}

${ }^{*}$ The different upper-case letters in the same row show the differences between stations $(p<0.05),{ }^{*}$ The different lower-case letters in the same column show the differences between months $(p<0.05)$ 
Table 4. Pearson correlation matrix for the heavy metals and major elements, OM, TP, TN, TOC in first station in July 2013.

\begin{tabular}{|c|c|c|c|c|c|c|c|c|c|c|c|c|c|}
\hline & $\mathrm{OM}$ & $\mathrm{TP}$ & TN & TOC & $\mathrm{Fe}$ & $\mathrm{Cu}$ & $\mathrm{Cd}$ & $\mathrm{Pb}$ & $\mathrm{Cr}$ & $\mathrm{Zn}$ & As & $\mathrm{Mn}$ & $\mathrm{Ni}$ \\
\hline $\mathrm{Ni}$ & 0.943 & 0.698 & -0.442 & -0.595 & -0.611 & -0.265 & 0.895 & -0.720 & -0.286 & $-0.954^{*}$ & -0.591 & -0.291 & - \\
\hline $\mathrm{Mn}$ & -0.593 & -0.691 & -0.606 & -0.439 & -0.575 & -0.232 & -0.011 & 0.795 & -0.832 & 0.093 & 0.856 & - & \\
\hline As & -0.800 & $-0.965^{*}$ & -0.461 & -0.287 & -0.231 & -0.414 & -0.479 & 0.741 & -0.538 & 0.512 & - & & \\
\hline $\mathrm{Zn}$ & -0.838 & -0.683 & 0.463 & 0.580 & 0.716 & 0.107 & $-0.987^{*}$ & 0.494 & 0.442 & - & & & \\
\hline $\mathrm{Cr}$ & 0.050 & 0.321 & 0.890 & 0.813 & 0.933 & 0.444 & -0.481 & -0.359 & - & & & & \\
\hline $\mathrm{Pb}$ & -0.879 & -0.651 & 0.000 & 0.196 & -0.016 & 0.290 & -0.366 & - & & & & & \\
\hline $\mathrm{Cd}$ & 0.761 & 0.674 & -0.426 & -0.522 & -0.722 & 0.008 & - & & & & & & \\
\hline $\mathrm{Cu}$ & -0.131 & 0.436 & 0.787 & 0.807 & 0.490 & - & & & & & & & \\
\hline $\mathrm{Fe}$ & -0.313 & 0.016 & 0.912 & 0.907 & - & & & & & & & & \\
\hline TOC & -0.342 & 0.155 & $0.980^{*}$ & - & & & & & & & & & \\
\hline $\mathrm{TN}$ & -0.155 & 0.316 & - & & & & & & & & & & \\
\hline $\mathrm{TP}$ & 0.834 & - & & & & & & & & & & & \\
\hline OM & - & & & & & & & & & & & & \\
\hline
\end{tabular}

${ }^{*}$ Correlation is statistically significant according to 0.05 level (two way). ${ }^{*}$ Correlation is statistically significant according to 0.01 level (two way).

Table 5. Pearson correlation matrix for the heavy metals and major elements, OM, TP, TN, TOC in second station in July 2013.

\begin{tabular}{|c|c|c|c|c|c|c|c|c|c|c|c|c|c|}
\hline & $\mathrm{OM}$ & $\mathrm{TP}$ & $\mathrm{TN}$ & TOC & $\mathrm{Fe}$ & $\mathrm{Cu}$ & $\mathrm{Cd}$ & $\mathrm{Pb}$ & $\mathrm{Cr}$ & $\mathrm{Zn}$ & As & Mn & $\mathrm{Ni}$ \\
\hline $\mathrm{Ni}$ & 0.268 & -0.946 & -0.507 & -0.293 & -0.452 & 0.430 & 0.293 & 0.507 & 0.112 & 0.267 & 0.202 & 0.924 & - \\
\hline $\mathrm{Mn}$ & 0.595 & -0.844 & -0.191 & -0.181 & -0.076 & 0.583 & 0.636 & 0.798 & 0.215 & 0.611 & 0.542 & - & \\
\hline As & 0.789 & -0.218 & 0.717 & -0.138 & 0.717 & 0.293 & $0.966^{*}$ & 0.877 & 0.595 & $0.984^{*}$ & - & & \\
\hline $\mathrm{Zn}$ & 0.884 & -0.225 & 0.625 & 0.004 & 0.719 & 0.460 & $0.997^{* *}$ & 0.943 & 0.452 & - & & & \\
\hline $\mathrm{Cr}$ & -0.018 & -0.381 & 0.598 & -0.845 & 0.160 & -0.534 & 0.383 & 0.243 & - & & & & \\
\hline $\mathrm{Pb}$ & 0.937 & -0.391 & 0.333 & 0.115 & 0.540 & 0.686 & $0.962^{*}$ & - & & & & & \\
\hline $\mathrm{Cd}$ & 0.917 & -0.225 & 0.577 & 0.067 & 0.713 & 0.530 & - & & & & & & \\
\hline $\mathrm{Cu}$ & 0.804 & -0.130 & -0.223 & 0.694 & 0.277 & - & & & & & & & \\
\hline $\mathrm{Fe}$ & 0.702 & 0.514 & 0.850 & 0.389 & - & & & & & & & & \\
\hline TOC & 0.437 & 0.586 & -0.115 & - & & & & & & & & & \\
\hline TN & 0.367 & 0.391 & - & & & & & & & & & & \\
\hline $\mathrm{TP}$ & -0.080 & - & & & & & & & & & & & \\
\hline OM & - & & & & & & & & & & & & \\
\hline
\end{tabular}

${ }^{*}$ Correlation is statistically significant according to 0.05 level (two way). ${ }^{* *}$ Correlation is statistically significant according to 0.01 level (two way).

It is important to determine whether concentrations of heavy metals in sediment pose a threat to aquatic organisms. The extent of metal pollution was assessed by comparing metal concentrations in the surface sediments at the stations in this study to the TEL/PEL and Target Limit values of some SQGs (MacDonald 2000) (Table 7 and Table 8). As shown in Table 8, in general, metal concentrations were lower than the average continental crust and average shale values except for $\mathrm{Pb}$ and $\mathrm{Cd}$ concentrations. The results of the calculation of Enrichment Factor and Igeo in Trabzon Harbour sediments are shown in Table 9. 
Table 6. Pearson correlation matrix for the heavy metals and major elements, OM, TP, TN, TOC in third station in July 2013.

\begin{tabular}{|c|c|c|c|c|c|c|c|c|c|c|c|c|c|}
\hline & OM & $\mathrm{TP}$ & TN & TOC & $\mathrm{Fe}$ & $\mathrm{Cu}$ & $\mathrm{Cd}$ & $\mathrm{Pb}$ & $\mathrm{Cr}$ & $\mathrm{Zn}$ & As & $\mathrm{Mn}$ & $\mathrm{Ni}$ \\
\hline $\mathrm{Ni}$ & 0.540 & -0.501 & -0.181 & -0.793 & -0.380 & -0.792 & -0.592 & -0.308 & 0.816 & 0.927 & -0.395 & $-0,922^{\star *}$ & - \\
\hline $\mathrm{Mn}$ & -0.438 & 0.491 & 0.273 & 0.822 & 0.270 & 0.864 & 0.514 & 0.183 & -0.884 & $-0.968^{\star}$ & 0.468 & - & \\
\hline As & 0.499 & -0.437 & -0.009 & 0.097 & -0.629 & 0.687 & 0.305 & -0.466 & -0.644 & -0.598 & - & & \\
\hline $\mathrm{Zn}$ & 0.213 & -0.435 & -0.421 & -0.832 & -0.035 & $-0.963^{*}$ & -0.345 & 0.072 & $0.973^{*}$ & - & & & \\
\hline $\mathrm{Cr}$ & 0.018 & -0.406 & -0.569 & -0.823 & 0.157 & $-0.998^{\star *}$ & -0.141 & 0.269 & - & & & & \\
\hline $\mathrm{Pb}$ & -0.894 & 0.232 & -0.575 & 0.000 & 0.920 & -0.337 & 0.697 & - & & & & & \\
\hline $\mathrm{Cd}$ & -0.522 & -0.174 & -0.683 & 0.000 & 0.456 & 0.135 & - & & & & & & \\
\hline $\mathrm{Cu}$ & 0.038 & 0.352 & 0.556 & 0.788 & -0.213 & - & & & & & & & \\
\hline $\mathrm{Fe}$ & $-0.984^{*}$ & 0.585 & -0.217 & 0.293 & - & & & & & & & & \\
\hline TOC & -0.426 & 0.853 & 0.717 & - & & & & & & & & & \\
\hline TN & 0.153 & 0.663 & - & & & & & & & & & & \\
\hline $\mathrm{TP}$ & -0.636 & - & & & & & & & & & & & \\
\hline OM & - & & & & & & & & & & & & \\
\hline
\end{tabular}

${ }^{*}$ Correlation is statistically significant according to 0.05 level (two way). ${ }^{* *}$ Correlation is statistically significant according to 0.01 level (two way).

Table 7. Comparison between metal concentrations (in $\mu \mathrm{g} \cdot \mathrm{g}^{-1} \mathrm{DW}$ ) determined in sediments from studied stations and the TEL, PEL and Target-Limit values of some SQGs [9].

\begin{tabular}{|c|c|c|c|c|c|c|c|c|}
\hline Heavy metal Content & Stations & As & $\mathrm{Cd}$ & $\mathrm{Cr}$ & $\mathrm{Cu}$ & $\mathrm{Pb}$ & $\mathrm{Ni}$ & $\mathrm{Zn}$ \\
\hline & I & $2.8-3.5$ & $0.07-0.16$ & $9.2-16.2$ & $5.8-7.1$ & $4.7-7.2$ & $9.3-20.1$ & $4.6-5.4$ \\
\hline \multirow[t]{2}{*}{ Trabzon Harbour } & II & $2.7-6.2$ & $0.11-0.23$ & $12.8-20.1$ & $10.5-14.8$ & $6.4-55.1$ & $11.4-54.2$ & $20.3-30.0$ \\
\hline & III & $3.5-6.3$ & $0.14-0.23$ & $9.4-28.6$ & $11.5-14.4$ & $5.7-14.3$ & $13.5-47.4$ & $27.1-54.3$ \\
\hline SQGs & & As & $\mathrm{Cd}$ & $\mathrm{Cr}$ & $\mathrm{Cu}$ & $\mathrm{Pb}$ & $\mathrm{Ni}$ & $\mathrm{Zn}$ \\
\hline \multicolumn{9}{|l|}{ Canadian } \\
\hline TEL & & 7.2 & 0.7 & 52.3 & 18.7 & 30.2 & 15.9 & 124 \\
\hline PEL & & 41.6 & 4.2 & 160 & 108 & 112 & - & 271 \\
\hline \multicolumn{9}{|l|}{ Wiskonsin } \\
\hline TEL & & 9.8 & 0.99 & 43 & 32 & 36 & 23 & 120 \\
\hline PEL & & 33 & 5.0 & 110 & 150 & 130 & 49 & 460 \\
\hline \multicolumn{9}{|l|}{ Flemish } \\
\hline Target value & & 20 & 2.5 & 60 & 20 & 70 & 70 & 160 \\
\hline Limit value & & 100 & 7 & 220 & 100 & 350 & 280 & 500 \\
\hline
\end{tabular}

Table 8. Comparison between metal concentrations (in $\mu \mathrm{g} \cdot \mathrm{g}^{-1} \mathrm{DW}$ ) determined in sediments from studied stations and the TEL and PEL-SQGs, Average Continental Crust, Average Shale and SQGs (Low-High).

\begin{tabular}{|c|c|c|c|c|c|c|c|c|}
\hline Heavy metal Content & Stations & As & $\mathrm{Cd}$ & $\mathrm{Cr}$ & $\mathrm{Cu}$ & $\mathrm{Pb}$ & $\mathrm{Ni}$ & $\mathrm{Zn}$ \\
\hline & I & $2.8-3.5$ & $0.07-0.16$ & $9.2-16.2$ & $5.8-7.1$ & $4.7-7.2$ & $9.3-20.1$ & $4.6-5.4$ \\
\hline \multirow[t]{2}{*}{ Trabzon Harbour } & II & $2.7-6.2$ & $0.11-0.23$ & $12.8-20.1$ & $10.5-14.8$ & $6.4-55.1$ & $11.4-54.2$ & $20.3-30.0$ \\
\hline & III & $3.5-6.3$ & $0.14-0.23$ & $9.4-28.6$ & $11.5-14.4$ & $5.7-14.3$ & $13.5-47.4$ & $27.1-54.3$ \\
\hline \multicolumn{2}{|l|}{ SQGs } & As & $\mathrm{Cd}$ & $\mathrm{Cr}$ & $\mathrm{Cu}$ & $\mathrm{Pb}$ & $\mathrm{Ni}$ & $\mathrm{Zn}$ \\
\hline \multicolumn{2}{|l|}{ TEL $^{1}$} & 7.24 & 0.68 & 52.3 & 18.7 & 30.2 & 15.9 & 124 \\
\hline \multicolumn{2}{|l|}{ PEL $^{1}$} & 41.6 & 4.21 & 160.0 & 108.0 & 112.0 & 42.8 & 271.0 \\
\hline \multicolumn{2}{|c|}{ Average Continental Crust ${ }^{2}$} & - & 0.1 & 35 & 25 & 20 & - & 71 \\
\hline \multicolumn{2}{|c|}{ Average Shale ${ }^{3}$} & - & 0.8 & 90 & 45 & 20 & 68 & 95 \\
\hline \multicolumn{2}{|c|}{ SQGs (Low-High) ${ }^{4}$} & $20-70$ & $1.5-10$ & $80-370$ & $65-270$ & $50-220$ & $21-52$ & $200-400$ \\
\hline
\end{tabular}

${ }^{1} \mathrm{TEL}$, threshold effect level, ${ }^{1} \mathrm{PEL}$, probable effect level [12]; ${ }^{2}$ Average Continental Crust; ${ }^{3}$ Average Shale; ${ }^{4} \mathrm{SQGs}$ (Low-High) [24]. 
Table 9. Seasonal and spatial variations of EF and Igeo values in Trabzon Harbour.

\begin{tabular}{|c|c|c|c|c|c|c|c|}
\hline \multirow{2}{*}{ Parameters } & \multirow{2}{*}{$\begin{array}{l}\text { Stations } \\
\text { Months }\end{array}$} & \multicolumn{2}{|c|}{ I } & \multicolumn{2}{|c|}{ II } & \multicolumn{2}{|c|}{ III } \\
\hline & & EF & Igeo & $\mathrm{EF}$ & Igeo & EF & Igeo \\
\hline \multirow{4}{*}{$\mathrm{Fe}$} & April 2013 & 14.49 & 3.27 & 29.06 & 4.28 & 25.45 & 4.09 \\
\hline & July 2013 & 17.76 & 3.57 & 27.67 & 4.21 & 27.77 & 4.21 \\
\hline & October 2013 & 14.13 & 3.24 & 23.95 & 4.00 & 20.70 & 3.79 \\
\hline & January 2014 & 15.49 & 3.37 & 24.04 & 3.79 & 20.73 & 3.79 \\
\hline \multirow{4}{*}{$\mathrm{Cu}$} & April 2013 & 0.16 & -3.28 & 0.33 & -2.21 & 0.36 & -2.10 \\
\hline & July 2013 & 0.18 & -3.08 & 0.26 & -2.51 & 0.35 & -2.10 \\
\hline & October 2013 & 0.15 & -3.37 & 0.37 & -2.03 & 0.29 & -2.39 \\
\hline & January 2014 & 0.15 & -3.31 & 0.37 & -2.02 & 0.36 & -2.08 \\
\hline \multirow{4}{*}{$\mathrm{Pb}$} & April 2013 & 0.27 & -2.46 & 0.38 & -1.99 & 0.34 & -2.16 \\
\hline & July 2013 & 0.39 & -1.94 & 0.73 & -1.05 & 0.84 & -0.84 \\
\hline & October 2013 & 0.42 & -1.82 & 0.60 & -1.31 & 0.51 & -1.56 \\
\hline & January 2014 & 0.32 & -2.23 & 3.24 & -1.11 & 0.70 & -1.10 \\
\hline \multirow{4}{*}{$\mathrm{Zn}$} & April 2013 & 0.07 & -4.41 & 0.42 & -1.83 & 0.84 & -0.84 \\
\hline & July 2013 & 0.08 & -4.31 & 0.31 & -2.27 & 0.61 & -1.31 \\
\hline & October 2013 & 0.08 & -4.31 & 0.46 & -1.70 & 0.42 & -1.85 \\
\hline & January 2014 & 0.08 & -4.18 & 0.45 & -1.73 & 0.47 & 0.32 \\
\hline \multirow{4}{*}{ Mn } & April 2013 & 0.042 & -5.14 & 0.042 & -5.15 & 0.47 & -5.01 \\
\hline & July 2013 & 0.040 & -5.22 & 0.041 & -5.18 & 0.044 & -5.10 \\
\hline & October 2013 & 0.031 & -5.59 & 0.061 & -4.62 & 0.044 & -5.09 \\
\hline & January 2014 & 0.035 & -5.43 & 0.060 & -4.65 & 0.052 & -4.86 \\
\hline \multirow{4}{*}{$\mathrm{Cr}$} & April 2013 & 0.13 & -3.59 & 0.18 & -3.05 & 0.13 & -3.56 \\
\hline & July 2013 & 0.16 & -3.22 & 0.22 & -2.79 & 0.39 & -1.95 \\
\hline & October 2013 & 0.17 & -3.16 & 0.17 & -3.12 & 0.17 & -3.14 \\
\hline & January 2014 & 0.22 & -2.78 & 0.27 & -2.47 & 0.23 & -2.69 \\
\hline
\end{tabular}

\section{Discussion}

In marine ecosystems, sediments act not only as carriers but also as a sink for contaminants, and they reflect the history of pollution in aquatic systems. The accumulation of certain trace metals generally occurs in the surface portion of the sediment by means of biological-geochemical mechanisms and becomes toxic to living aquatic organisms. Along the coastline of the Black Sea, industry has been developing intensively and quickly. In other words, there are many plants (iron and steel), power stations and oil refineries. The waste-water from plants, atmospheric deposition, the metallurgical industry, the discharge of pollutants from shipping vehicles and transport, and especially mining waste, are possible anthropogenic sources of metals in the sediment. As there are only a limited number of studies [14] [15] [16] [17] on heavy metal concentrations in the sediment of the Black Sea coastline, this research conducted in Trabzon Harbour aims to reveal the anthropogenic effects of metals and the environmental risk associated with metal concentrations. The concentration of heavy metals in the surface sediment had the trend $\mathrm{Fe}>\mathrm{Pb}>\mathrm{Zn}>\mathrm{Ni}>\mathrm{Mn}>\mathrm{Cr}>\mathrm{Cu}>\mathrm{As}>\mathrm{Cd}$. 
The maximum concentration values for $\mathrm{Fe}, \mathrm{Cd}, \mathrm{Pb}$ and $\mathrm{Ni}$ in the sediment samples at the Trabzon Harbour stations were higher than the findings that reported by the researchers above. On the other hand, the sediment $\mathrm{Cu}, \mathrm{Cr}, \mathrm{Zn}$, and $\mathrm{Mn}$ concentrations determined in this study were generally lower than the results of [14] [15] [25] [26]. Trabzon Harbour seems to be affected by the heavy atmospheric rains in the Eastern Black Sea Region, the mining facilities and the sediment's geological structure as well. The magmatic rocks of the northern part of Turkey, rich in mineral deposits, contribute significant amounts of $\mathrm{Cr}, \mathrm{Ni}, \mathrm{Cu}$, $\mathrm{Pb}$ and $\mathrm{Zn}$ to this region's sediment.

In estuaries, where, in addition to natural inputs, concomitant activities such as harbour activities and industrial, agricultural and residential activities can cause heavy metal release from the sediment to the environment. In the Eastern Black Sea Region, residents and the industrial sector generally use coal or fuel oil for heating. It is well known that ash particles occur as a result of fossil fuel consumption, and this ash is a potential effective source for the atmospherical discharge of many metals. The higher concentrations of $\mathrm{Zn}, \mathrm{Cu}, \mathrm{Mn}, \mathrm{Ni}, \mathrm{As}$ and $\mathrm{Pb}$, especially in the fall and winter, show that fossil fuel consumption plays a major role in metal composition management in this study.

Chlorophyll-a concentrations reached their maximum levels in the spring and fall periods [27]. The higher concentrations of Mn in the sediment in this study were found at the first and second stations in April and October, revealing that there was a relation between the water column and the sediment. This coincides with the association of the phytoplankton bloom in fall and spring with the maximum levels of manganese in the sediment.

Sediment metal concentrations were evaluated as $\mathrm{Cd}: 0.58 \mathrm{mg} \cdot \mathrm{kg}^{-1}$ and $\mathrm{Zn}$ : $596 \mathrm{mg} \cdot \mathrm{kg}^{-1}$ in Taiwan Kaohsiung Port between 2002 and 2005 [8]. The maximum concentrations of $\mathrm{Cd}$ and $\mathrm{Zn}$ were determined in October as $0.23 \mathrm{mg} \cdot \mathrm{kg}^{-1}$ at the second station and $54 \mathrm{mg} \cdot \mathrm{kg}^{-1}$ at the third station. The maximum $\mathrm{Zn}$ concentration in Trabzon Harbour was found to be lower, whereas the highest Cd concentration was close to the above researcher's findings. It is a well-known fact that Zn, a key and essential element for all living things, has a lower toxicity when compared to other metals. Therefore, $\mathrm{Zn}$ concentrations were not found to be high enough to pose a threat to the aquatic organisms in the Trabzon Harbour.

The distribution of some heavy metals ( $\mathrm{Cu}, \mathrm{Zn}, \mathrm{Cd}, \mathrm{Pb}, \mathrm{Fe}, \mathrm{Ni}, \mathrm{Cr}$ and $\mathrm{Mn}$ ) in the sediment of the coastline of southwestern Spain was investigated by [28]. The maximum concentrations of certain heavy metals there were ( $\mathrm{Zn}: 649 \mathrm{mg}$. $\mathrm{kg}^{-1}, \mathrm{Cu}: 336 \mathrm{mg} \cdot \mathrm{kg}^{-1}, \mathrm{~Pb}: 197 \mathrm{mg} \cdot \mathrm{kg}^{-1}$, and $\mathrm{Cd}: 2.5 \mathrm{mg} \cdot \mathrm{kg}^{-1}$ ), evaluated over a $35-\mathrm{km}$ length along the coastline close to the region where the mouths of the Tinto and Odiel rivers combine. According to our study conducted in Trabzon Harbour, contrary to the sediment $\mathrm{Ni}$ concentration, $\mathrm{Fe}, \mathrm{Cr}, \mathrm{Cu}$ and $\mathrm{Pb}$ concentrations were found to be much lower than the researches indicated above. This could be a result of the lower number of industrial facilities near Trabzon Harbour. A positive correlation was assessed between Fe and Cd in Trabzon Har- 
bour in April, October and January, and $\mathrm{Zn}$ and $\mathrm{Cd}$ in July and January. This positive correlation triggers the flux of $\mathrm{Zn}$ into the aquatic system moving with the effluents following the industrial production of $\mathrm{Zn}, \mathrm{Cu}, \mathrm{Pb}$ and $\mathrm{Fe}$.

Organic matter in colloidal form plays an important role in the exchange capacity of sediment, whereas in the presence of iron, organic matter adsorbs greater amounts of phosphates, as these carry negative surface charges. A high sediment organic material content ensures a fractional humic material context; therefore, this can play a critical role in the phosphorus retention mechanism [29]. The sediment organic material concentration was detected as $<20 \%$ spatially and seasonally at Trabzon Port, and the positive correlation between TP and TOC indicated phosphorus retention in the sediment.

In aquatic ecosystems, total organic carbon is an important factor in controlling the abundance of some heavy metals [30]. A positive correlation was established between TOC and TP and also between TOC and some heavy metals ( $\mathrm{Pb}$, $\mathrm{Cu}$ and $\mathrm{Fe}$ ) in July at Trabzon Port. The positive correlation between the sediment total organic carbon and the heavy metals in Trabzon Port was compatible with [5] [30] [31], who stated that TOC is the key element in controlling the abundance of heavy metals in sediment.

The degree to which the heavy metal load from the sediment is toxic and harmful to the aquatic environment depends on the geochemical conditions of the sediment. Furthermore, the assessment of contamination status is based mainly on SQGs or quantitative indices such as the geo accumulation index (Igeo) and Enrichment Factor. According to the Canadian and Wisconsin SQGs, there are three ranges for TEL and PEL values as follows: There is no adverse biological effect if the concentrations of metals in the sediment are lower than the TEL values. When the values are between TEL and PEL values, this may occasionally be associated with an adverse biological effect. If the concentrations are higher than PEL values, there is an association with adverse biological effects [32].

According to Flemish SQGs for dredged materials [33]: 1) The dredged sediment may be dumped at sea when metal concentrations are lower than the target value. 2) Further investigation is needed when the concentrations are between the target and limit values. 3) Dumping the dredged sediment into the sea is forbidden if the concentrations are higher than the limit value. The comparison results of Trabzon Port and the Flemish SQGs are given in Table 4. Sediment heavy metal values of Trabzon Port are all lower than the target values, indicating that the dredged sediment may be dumped at sea [9].

TEL/PEL SQGs are applied to determine the degree to which the sediment-associated chemical status might adversely affect the aquatic organisms. The threshold effects level is intended to present chemical concentrations below which adverse biological effects rarely occur, and the probable effects level is intended to present chemical concentrations above which adverse biological effects frequently occur [12]. The heavy metal concentrations in this study were compared with the TEL and PEL values, and the results are presented in Table 7 and 
Table 8 [9]. Among the investigated elements, $\mathrm{Pb}$ and Ni most often exceeded the TEL and PEL values, and so were expected to have occasional adverse biological effects. Therefore, the contamination ranking of the $\mathrm{Pb}$ and $\mathrm{Ni}$ loads in the sediment compared with regional background levels seems to be reliable, and this result is in agreement with [34]. The sediment $\mathrm{Pb}$ (second station) value exceeded the values with respect to the Average Continental Crust, Average Shale and SQGs (Low-High) [24], and Ni (second and third stations) values only exceeded the SQGs (Low-High) value.

Concentrations of eight heavy metals in the surface sediment of the western part of the Egyptian Mediterranean Coast were assessed to evaluate the concentration levels and spatial distributions with the Threshold Effect Concentration (TEC) and Probable Effect Concentration (PEC) by [35]. There was not an adverse biological effect when sediment heavy metal concentrations were lower than the TEC, whereas there was a significant risk if the concentrations were greater than the PEC level. Sediment heavy metals $(\mathrm{Cd}, \mathrm{Cr}, \mathrm{Pb}, \mathrm{Cu}$ and $\mathrm{Zn}$ ) in Trabzon Port were in line with the findings of [35], who stated that there is no risk to aquatic organisms at these levels. However, $\mathrm{Ni}$, the main source of minerals, reached its maximum value $\left(54.2 \mathrm{mg} \cdot \mathrm{kg}^{-1}\right)$ at the second station in January. With the increase of heavy rain in this period, river discharge and erosion also increase, and this leads to increasing amounts of Ni in Trabzon Port. To assess the metal contamination in the surface sediment of Trabzon Port, the concentrations of the most toxic metals ( $\mathrm{As}, \mathrm{Cd}, \mathrm{Cr}, \mathrm{Cu}, \mathrm{Pb}, \mathrm{Ni}$ and $\mathrm{Zn}$ ) were compared with USEPA data. According to the USEPA sediment guideline [10] [11], the maximum sediment As concentration (2.7 - $\left.6.3 \mu \mathrm{g} \cdot \mathrm{g}^{-1} \mathrm{DW}\right)$ was considered moderately polluting $(3-8)$, the $\mathrm{Cr}$ concentration ( $\max$ value $28.6 \mu \mathrm{g} \cdot \mathrm{g}^{-1} \mathrm{DW}$ ) was evaluated as moderately polluting $(25-75)$ and $\mathrm{Pb}\left(55 \mu \mathrm{g} \cdot \mathrm{g}^{-1} \mathrm{DW}\right)$ was also at the same pollution level $(40-60)$. $\mathrm{Ni}\left(\max 54.2 \mu \mathrm{g} \cdot \mathrm{g}^{-1} \mathrm{DW}\right)$ was moderately polluting at the third station, but heavily polluting at the second station. The maximum $\mathrm{Cu}$ concentration $\left(5.8-14.8 \mu \mathrm{g} \cdot \mathrm{g}^{-1} \mathrm{DW}\right)$ at $<25$ was non-polluting, and $\mathrm{Zn}\left(4.6-54.3 \mu \mathrm{g} \cdot \mathrm{g}^{-1} \mathrm{DW}\right)<90$ values were non-polluting as well. Possible anthropogenic sources of metals in the sediment of Trabzon Port are likely the discharge of untreated waste, commonly industrial and municipal, sewage run-off and petroleum contamination in the port. Especially $\mathrm{Ni}, \mathrm{Cu}, \mathrm{Pb}, \mathrm{Zn}$ and $\mathrm{Cr}$ have been found to be associated with petroleum contamination in coastal environments, as [5] indicated.

According to [13] [36], contamination levels may be classified in the following order: Igeo $\leq 0=$ unpolluted, Igeo $<1=$ unpolluted to moderately polluted, Igeo $<2=$ moderately polluted, Igeo $<3=$ moderately to strongly polluted, Igeo $<4=$ strongly polluted, Igeo $<5=$ strongly to very strongly polluted, Igeo $>5=$ very strongly polluted. The results of the geoaccumulation index revealed that Trabzon Port, Igeo values for $\mathrm{Cu}, \mathrm{Pb}, \mathrm{Cr}, \mathrm{Zn}$ and $\mathrm{Mn}$ were lower than zero, indicating that sediment pollution level remained at a very low degree. In contrast, the Fe concentrations of between 3 and 4 at the control station shows moderate to strong pollution however, at the second and third stations, pollution levels were 
found to be strongly to very strongly polluted (between 4 and 5) in Trabzon Port.

According to [8], $\mathrm{EF}<1$ indicates no enrichment, $\mathrm{EF}<3$ is minor enrichment, $\mathrm{EF}=3-5$ is moderate enrichment, $\mathrm{EF}=5-10$ is moderately severe enrichment, $\mathrm{EF}=10-25$ is severe enrichment, $\mathrm{EF}=25-50$ is very severe enrichment, and EF>50 is extremely severe enrichment. The calculation of enrichment factors in Trabzon Port for the three stations showed that: EF values for $\mathrm{Cu}, \mathrm{Pb}, \mathrm{Cr}, \mathrm{Zn}, \mathrm{Mn}$ and $\mathrm{Cr}$ were $<1$, showing low pollution levels, whereas $\mathrm{EF}$ values for $\mathrm{Fe}$ concentration at the three stations demonstrated a moderately severe enrichment pollution level at $>6$.

\section{Conclusion}

Although there are a limited number of existing studies on the sediment of the Black Sea coastline, there has been no research revealing the anthropogenical effect of metals in Trabzon Port. This study investigated the magnitude and ecological relevance of anthropogenic heavy metal pollution in Trabzon Port by means of sediment quality assessment methods. Comparison with sediment quality guidelines revealed that $\mathrm{Fe}$ and $\mathrm{Pb}$ in the Port sediment are the two main threats causing adverse biological effects. In general, relatively higher ecotoxicological potentials were found at the port stations than at the control station. In conclusion, in addition to natural inputs, concomitant activities such as harbour activities within the port and nearby industrial, agricultural and mining activities can release heavy metals, which could soon pose a threat to the environment due to their accumulation in the sediment. The data from this study regarding metal levels should be used as a baseline reference for future metal pollution monitoring programs in Trabzon Port, which will be a useful tool for authorities in charge of sustainable management.

\section{References}

[1] Adeyemo, O.K., Adedokun, O.A., Yusuf, R.K. and Adeleye, E.A. (2008) Seasonal Changes in Physico-Chemical Parameters and Nutrient Load of River Sediment in Ibadan City, Nigeria. Global NEST Journal, 10, 326-336.

[2] Davies, O.A. and Abowei, J.F.N. (2009) Sediment Quality of Lower Reaches of Okpoka Creek, Niger Delta, Nigeria. European Journal of Scientific Research, 26, 437442.

[3] Rizo, O.D., Rudnikas, A.G., Perez, R.D.L., Caraballo, G.A. and Rodriguez, K.A. (2014) XRF Analysis of Sediments from Nuevitas Bay (Cuba): Assessment of Current Heavy Metal Contamination. Nucleus, 55, 11-14.

[4] Praveena, S.M., Radojevic, M., Abdullah, M.H. and Avis, A.Z. (2007) Factor-Cluster Analysis and Enrichment Study of Mangrove Sediments-An Example from Mengkabong Sabah. The Malysian Journal of Analytical Sciences, 2, 421-430.

[5] Wang, X.-C., Feng, H., Hai-Qing, M. (2007) Assessment of Metal Contamination in Surface Sediments of Jiaozhou Bay, Qingdao, China. Clean Soil Air Water, 35, 6270. https://doi.org/10.1002/clen.200600022

[6] Akinmosin, A., Osinowo, O.O. and Oladunjoye, M.A. (2009) Radiogenic Components of the Nigeria Tarsand Deposits. Earth Sciences Research Journal, 13, 64-73. 
[7] MacDonald, D.D., Carr, R.S., Calder, F.D., Long, E.R. and Ingersoll, C.G. (1996) Development and Evaluation of Sediment Quality Guidelines for Florida Coastal Waters. Ecotoxicology, 5, 253-278. https://doi.org/10.1007/BF00118995

[8] Chen, C.W., Kao, C.M., Chen, C.F. and Dong, C.D. (2007) Distribution and Accumulation of Heavy Metals in the Sediments of Kaohsiung Harbor, Taiwan. Chemosphere, 66, 1431-1440.

[9] Ho, H.H., Swennen, R. and Damme, A.V. (2010) Distribution and Contamination Status Of Heavy Metals in Estuarine Sediments near Cua Ong Harbor, Ha Long Bay Vietnam. Geologica Belgica, 13, 37-47.

[10] USEPA (1996) SW-846 Reference Methodology: Method 6010B. Inductively Coupled Plasma-Atomic Emission Spectrometry.

[11] USEPA (1999) SW-846 Reference Methodology: Method 3050B. Standard Operating Procedure for the Digestion of Soil/Sediment Samples Using a Hotplate/Beaker Digestion Technique.

[12] MacDonald, D.D., Ingersoll, C.G. and Berger, T.A. (2000) Development and Evaluation of Consensus-Based Sediment Quality Guidelines for Freshwater Ecosystems. Archives of Environmental and Contamination Toxicology, 39, 20-31. https://doi.org/10.1007/s002440010075

[13] Gonzales-Macias, C., Schifter, I., Lluch-Cota, D.B., Mendez-Rodriguez, L. and Hernandez-Vazquez, S. (2006) Distrubution, Enrichment and Accumulation of Heavy Metals in Coastal Sediments of Salina Cruz Bay, Mexico. Environmental Monitoring and Assessment, 118, 211-230. https://doi.org/10.1007/s10661-006-1492-8

[14] Topçuoğlu, S., Kırbaşoğlulu, C. and Güngör, N. (2002) Heavy Metals in Organisms and Sediments from Turkish Coast of the Black Sea, 1997-1998. Environmental International, 27, 521-526.

[15] Ergül, H.A., Topçuoğlu, S., Ölmez, E. and Kırbaşoğlu, Ç. (2008) Heavy Metals in Sinking Particles and Bottom Sediments from the Eastern Turkish Coast of the Black Sea. Estuarine, Coastal and Shelf Science, 78, 396-402.

[16] Boran, M. and Altınok, İ. (2010) A Review of Heavy Metals in Water, Sediment and Living Organisms in the Black Sea. Turkish Journal of Fisheries and Aquatic Sciences, 10, 565-572. https://doi.org/10.4194/trifas.2010.0418

[17] Alkan, N., Alkan, A., Akbaş, U. and Fisher, A. (2015) Metal Pollution Assessment in Sediments of the Southeastern Black Sea Coast of Turkey. Soil and Sediment Contamination: An International Journal, 24, 290-305. https://doi.org/10.1080/15320383.2015.950723

[18] Anonymous (2011) Talepler el kitabı-T.C. Başbakanlık Denizcilik Müsteşarlığg (Deniz Ticareti Genel Müdürlüğü).

[19] Anonymous (2009). http://al-port.com/Hakkimizda.aspx

[20] Anonymous (2013). http://al-port.com/Hakkimizda.aspx

[21] Kacar, B. (1995) Bitki ve Toprağın Kimyasal Analizleri 3: Toprak Analizleri Ankara Üniersitesi Ziraat Fakültesi Eğitim Araştırma ve Geliştirme Vakfı Yayınları, No: 3, 705 s. (in Turkish)

[22] Shrestha, M.K. and Lin, C.K. (1996) Determination of Phosphorus Saturation Level in Relation to Clay Content in Formulated Pond Muds. Aquacultural Engineering, 15, 441-459.

[23] Kesici, T. and Kocabaş, Z. (2007) Biyoistatistik, Ankara Üniv. Eczacılık Fakültesi Yayın No. 94, Ankara.

[24] Simpson, S.L., Batley, G.E., Chariton, A.A., Stauber, J.L., King, C.K., Chapman, J.C., 
Hyne, R.V., Gate, S.A., Roach, A.C. and Maher, W.A. (2005) Handbook for Sediment Quality Assesment. Bangor NSW, CSIRO.

[25] Topçuoğlu, S., Ölmez, E., Kırbaşoğlu, C., Yılmaz, Y.Z. and Saygın, N. (2004) Heavy Metal and Radioactivity in Biota and Sediment Samples Collected from Ünye in the Eastern Black Sea. Proceedings 37 th CIESM, Barcelona, 250 p.

[26] Balkis, N., Topçuoğlu, S., Güven, K.C., Öztürk, B., Topaloğlu, B., Kırbaşoğlu, Ç. and Aksu, A. (2007) Heavy Metals in Shallow Sediments from the BlackSea, Marmara Sea and Aegean Sea regions of Turkey. Journal of Black Sea/Mediterranean Environment, 13, 147-153.

[27] Alkan, A., Zengin, B. and Serdar, S. (2004) Determination Some Physical and Chemical Parameters of Seawater on the Trabzon Coast, Final Report (2001-2003). Water Product Central Research Institutes Publishing, Trabzon, 113 p. (in Turkish)

[28] Morillo, J., Usero, J. and Gracia, I. (2004) Heavy Metal Distribution in Marine Sediments from the Southwest Coast of Spain. Chemosphere, 55, 431-442.

[29] Istvanovics, V. (1994) Fractional Composition, Adsorption and Release of Sediment Phosphorus in the Kiss-Balaton Reservoir. Water Research, 28, 717-726.

[30] Li, G., Hu, B., Bi, J., Leng, Q., Xiao, C. and Yang, Z. (2013) Heavy Metals Distribution and Contamination in Surface Sediments of the Coastal Shandong Peninsula (Yellow Sea). Marine Pollution Bulletin, 76, 420-426.

[31] Çevik, F., Göksu, M.Z.L., Derici, O.B. and Fındık, Ö. (2009) An Assessment of Metal Pollution in Surface Sediments of Seyhan Dam by Using Enrichment Factor, Geoaccumulation Index and Statistical Analyses. Environmental Monit Assessment, 152, 309. https://doi.org/10.1007/s10661-008-0317-3

[32] MacDonald, D.D., Ingersoll, C.G., Dawn, E.S. and Rebecca, A.L. (2003) Development and Applications of Sediment Quality Criteria for Managing Contaminated Sediment in British Columbia. MacDonald Environmental Sciences Ltd., Island Highway North Nanaimo, British Columbia, V9T1W6.

[33] MUMM (2007) Management Unit of the North Sea Mathematical Models (MUMM)-Royal Belgian Institute of Natural Sciences 2007. Dredged Material. http://www.mumm.ac.be/EN/Management/Sea-based/dredging.php

[34] Li, J. (2014) Risk Assessment of Heavy Metals in Surface Sediments from the Yanghe River, China. International Journal of Environmental Research and Public Health, 11, 12441-12453. https://doi.org/10.3390/ijerph111212441

[35] Ahdy, H.H. and Khaled, A. (2009) Heavy Metals Contamination in Sediments of the Western Part of Egyptian Mediterranean Sea. Australian Journal of Basic and Applied Sciences, 3, 3330-3336.

[36] Loska, K. and Wiechula, D. (2003) Application of Principal Component Analysis for the Estimation of Source of Heavy Metal Contamination in surface Sediments from Rybnik Reservoir. Chemosphere, 51, 723-733. 
Submit or recommend next manuscript to SCIRP and we will provide best service for you:

Accepting pre-submission inquiries through Email, Facebook, LinkedIn, Twitter, etc. A wide selection of journals (inclusive of 9 subjects, more than 200 journals)

Providing 24-hour high-quality service

User-friendly online submission system

Fair and swift peer-review system

Efficient typesetting and proofreading procedure

Display of the result of downloads and visits, as well as the number of cited articles Maximum dissemination of your research work

Submit your manuscript at: http://papersubmission.scirp.org/

Or contact oje@scirp.org 